\title{
CHANGES IN PLUTO'S ATMOSPHERE: 1988-2006
}

\author{
J. L. Elliot, ${ }^{1,2,3}$ M. J. Person, ${ }^{1}$ A. A. S. Gulbis, ${ }^{1}$ S. P. Souza, ${ }^{4}$ E. R. Adams, ${ }^{1}$ B. A. Babcock, ${ }^{5}$ \\ J. W. Gangestad, ${ }^{4,6}$ A. E. Jaskot ${ }^{4}$ E. A. Kramer, ${ }^{1}$ J. M. Pasachoff, ${ }^{4}$ R. E. Pike, ${ }^{1}$ \\ C. A. Zuluaga, ${ }^{1}$ A. S. Bosh, ${ }^{7}$ S. W. Dieters, ${ }^{8}$ P. J. Francis, ${ }^{9}$ A. B. Giles, ${ }^{8}$ \\ J. G. Greenhill, ${ }^{8}$ B. Lade, ${ }^{10}$ R. Lucas, ${ }^{11}$ And D. J. Ramm ${ }^{12}$ \\ Received 2006 December 19; accepted 2007 March 8
}

\begin{abstract}
The 2006 June 12 occultation of the star P384.2 (2UCAC 26039859) by Pluto was observed from five sites in southeastern Australia with high-speed imaging photometers that produced time-series CCD images. Light curves were constructed from the image time series and fit by least-squares methods with model light curves. A new modeling procedure is presented that allows a simultaneous fit of the atmospheric parameters for Pluto and the astrometric parameters for the occultation to all of the light curves. Under the assumption of a clear atmosphere and using this modeling procedure to establish the upper atmosphere boundary condition, immersion and emersion temperature profiles were derived by inversion of the Siding Spring light curve, which had our best signal-to-noise ratio. Above $\sim 1230 \mathrm{~km}$ radius, atmospheric temperatures are $\sim 100 \mathrm{~K}$ and decrease slightly with altitude — the same as observed in 1988 and 2002 . Below $1210 \mathrm{~km}$, the temperature abruptly decreases with altitude (gradients $\sim 2.2 \mathrm{~K} \mathrm{~km}^{-1}$ ), which would reach the expected $\mathrm{N}_{2}$ surface-ice temperature of $\sim 40 \mathrm{~K}$ in the $1158-1184 \mathrm{~km}$ radius range. This structure is similar to that observed in 2002, but a much stronger thermal gradient (or stronger extinction) is implied by the 1988 light curve (which shows a "kink" or "knee" at $1210 \mathrm{~km}$ ). The temperature profiles derived from inversion of the present data show good agreement with a physical model for Pluto's atmosphere selected from those presented by Strobel et al. (1996). Constraints derived from the temperature profiles (and considering the possibility of a deep troposphere) yield a value of $1152 \pm 32 \mathrm{~km}$ for Pluto's surface radius. This value is compared with surface-radius values derived from the series of mutual occultations and eclipses that occurred in 1985-1989, and the limitations of both types of measurements for determining Pluto's surface radius are discussed. The radius of Pluto's atmospheric shadow at the half-intensity point is $1207.9 \pm 8.5 \mathrm{~km}$, the same as obtained in 2002 within measurement error. Values of the shadow radius cast by Pluto's atmosphere in 1988, 2002, and 2006 favor frost migration models in which Pluto's surface has low thermal inertia. Those models imply a substantial atmosphere when New Horizons flies by Pluto in 2015. Comparison of the shape of the stellar occultation light curves in 1988, 2002, and 2006 suggests that atmospheric extinction, which was strong in 1988 (15 months before perihelion), has been dissipating.
\end{abstract}

Key words: methods: data analysis — occultations — planets and satellites: individual (Pluto)

\section{INTRODUCTION}

Most of our knowledge of Pluto's atmosphere comes from stellar occultations observed in 1988 and 2002, during which the atmosphere was extensively probed by observations from multiple stations (Hubbard et al. 1988; Elliot et al. 1989, 2003b; Millis et al. 1993; Sicardy et al. 2003; Pasachoff et al. 2005). For the 1988 stellar occultation, the highest quality light curve was obtained by the Kuiper Airborne Observatory (KAO; $0.9 \mathrm{~m}$ tele-

\footnotetext{
${ }^{1}$ Department of Earth, Atmospheric, and Planetary Sciences, Massachusetts Institute of Technology, Cambridge, MA 02139-4307, USA.

2 Department of Physics, Massachusetts Institute of Technology, Cambridge, MA 02139-4307, USA.

3 Lowell Observatory, Flagstaff, AZ 86001, USA.

4 Astronomy Department, Williams College, Williamstown, MA 01267-2565, USA.

5 Physics Department, Williams College, Williamstown, MA 01267-2565, USA.

${ }^{6}$ Current address: Department of Aeronautics and Astronautics, Purdue University, West Lafayette, IN 47907, USA.

7 Department of Astronomy, Boston University, Boston, MA 02215; current address: Lowell Observatory, Flagstaff, AZ 86001, USA.

8 School of Mathematics and Physics, University of Tasmania, Hobart, TAS 7001, Australia.

9 Research School of Astronomy and Astrophysics, Australian National University, Mount Stromlo Observatory, Weston, ACT 2611, Australia.

10 Astronomical Society of South Australia, Inc., Adelaide, SA 5001, Australia.

11 School of Physics, University of Sydney, NSW 2006, Australia.

12 Department of Physics and Astronomy, University of Canterbury, Christchurch, New Zealand.
}

scope), which revealed an unexpected structure (Elliot et al. 1989). Although the upper part of the light curve followed the shape expected for a stellar occultation by an isothermal atmosphere, just below the half-light level the light curve exhibited a "kink" below which the flux dropped abruptly. The drop was well below the extrapolation of the isothermal model that fit the upper part of the light curve (see Fig. 3 of Elliot et al. 1989) and eventually reached a minimum value indistinguishable from zero (Elliot et al. 2003a).

Although the kink in the light curve could be explained either by extinction or by a decrease in scale height (proportional to temperature), the difficulties of achieving such a low stellar flux (consistent with zero) with refractive models led Elliot et al. (1989) to choose an extinction model for their initial analysis. Later refined by Elliot \& Young (1992), this model found that the upper part of the atmosphere probed by the occultation (at radii greater than $1215 \mathrm{~km}$ ) was nearly isothermal at $\sim 104 \mathrm{~K}$ (for an $\mathrm{N}_{2}$ atmosphere), with the abrupt onset of an extinction layer at a radius of $1215 \mathrm{~km}$. The scale height of the extinction was about $30 \mathrm{~km}$, half that of the isothermal atmosphere at higher altitude.

The occultation results from 1988 sparked several theoretical and observational efforts. Yelle \& Lunine (1989) found that the temperature of the isothermal part of Pluto's atmosphere agreed with that expected from radiative equilibrium, with a small amount of $\mathrm{CH}_{4}$ embedded in a predominantly heavier gas of molecular weight 28, which was later identified to be $\mathrm{N}_{2}$ (Owen et al. 1993). 
Stansberry et al. (1989) noted that the extinction required by the Elliot et al. (1989) model for the light curve could not be produced by $\mathrm{CH}_{4}$ photochemical processes known to be active on Titan, Uranus, and Neptune. Nor would the upper boundary of such $\mathrm{CH}_{4}$ photochemical hazes be expected to be abrupt.

Going beyond the refraction model originally considered by Elliot et al. (1989), Eshleman (1989) and Hubbard et al. (1990) proposed - as an alternative to the extinction model - refraction models that contained a thermal gradient. They pointed out that surface temperatures for Pluto would be $\sim 50-60 \mathrm{~K}$ below those found in the upper atmosphere. Hence, a thermal gradient must be present somewhere in the atmosphere to reach the surface temperature, but it remained unknown if the location of the thermal gradient is within the radius range probed by the occultation, or if the thermal gradient would be steep enough to produce an occultation light curve that would match the 1988 KAO data. Building on the work of Yelle \& Lunine (1989) and Lellouch (1994, who pointed out the importance of $\mathrm{CO}$ cooling in radiativeconductive models), Strobel et al. (1996) formulated a more complete radiative-conductive model that included a non-LTE treatment for solar heating in the 2.3 and $3.3 \mu \mathrm{m}$ bands of $\mathrm{CH}_{4}$, cooling by the $7.6 \mu \mathrm{m}$ band of $\mathrm{CH}_{4}$ and $\mathrm{CO}$ rational line emission. They could approach an explanation of the 1988 KAO light curve by invoking a region of strong turbulent $\mathrm{CH}_{4}$ mixing in the lower regions probed by the stellar occultation, immediately overlying a troposphere (Stansberry et al. 1994).

An observational approach for discriminating between refraction and extinction models for Pluto's atmosphere would be to observe an occultation at two or more well-separated wavelengths. Since extinction would have a much larger wavelength dependence than refraction, one would expect the shapes near the bottoms of the occultation light curves recorded at different wavelengths to be significantly different if extinction dominated the structure of the light curve. On the other hand, if the structure of the light curve were dominated by refraction effects, the light curves at different wavelengths would be virtually the same.

Multiwavelength observations were made at NASA's Infrared Telescope Facility (IRTF) and other telescopes at Mauna Kea, Hawaii, for a stellar occultation that occurred in 2002 (Elliot et al. 2003b; Pasachoff et al. 2005). The flux of the occultation light curves did not go to zero as it did for the 1988 occultation, leaving a residual stellar flux in the middle of the light curves. The occultation light curves spanned the wavelength range $0.75-2.2 \mu \mathrm{m}$, and the residual stellar flux showed a definite wavelength trend, with more at longer wavelengths (Elliot et al. 2003b), as would be expected from extinction by particles comparable in size to the wavelength. These observations showed that at least some extinction was present in Pluto's atmosphere in 2002.

An astrometric analysis was also performed on the 2002 multichord occultation data to determine the radius and pressure at the "half-light" level by Elliot et al. (2003b). They found that Pluto's atmospheric pressure (at a constant radius) increased by approximately a factor of 2 since the 1988 measurement (Millis et al. 1993). The same conclusion was reached by Sicardy et al. (2003) based on a single-chord observation of the same event (from the Canada-France-Hawaii Telescope), combined with the astrometric constraint imposed by the occultation prediction. Although such a change in atmospheric pressure can be explained by frost-migration models (Hansen \& Paige 1996), one must obtain a series of occultation observations - ideally every few years - in order to establish detailed atmospheric behavior. The largest changes should occur near perihelion, which was reached on 1989 September 5, approximately 15 months after the 1988 June 9 occultation was observed (Millis et al. 1993).

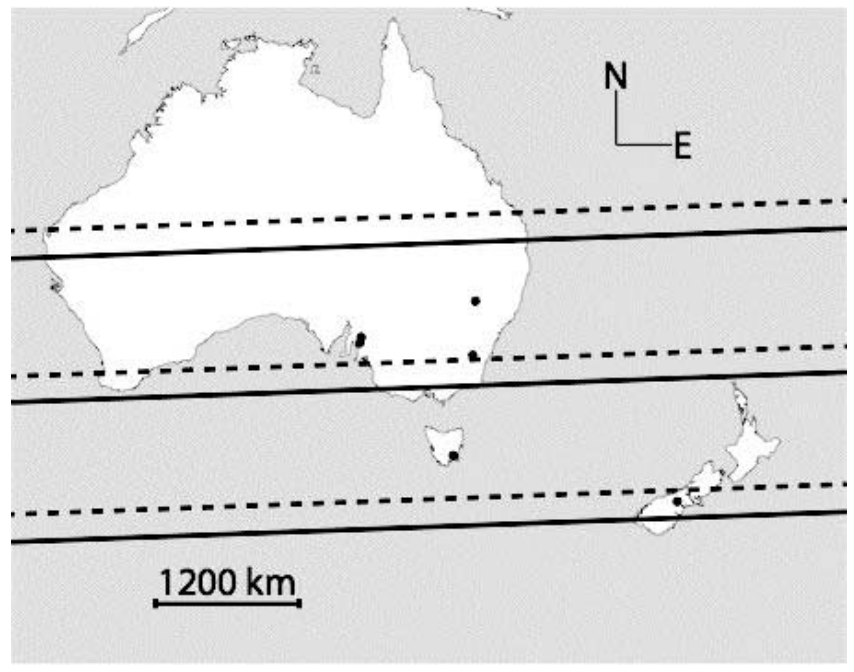

FIG. 1.-Location of observing sites. This localized map of the region where the Pluto occultation was observed shows the locations of the observing sites (black dots) given in Table 1. Also plotted is the location of Mount John, New Zealand, where observations were not successful (see text). The last prediction posted on the MIT Web site prior to the occultation (see footnote 13) is illustrated by the three dashed lines, which indicate, from top to bottom, the predicted northern limit of the shadow (assuming a radius of $1200 \mathrm{~km}$, which corresponds to a stellar flux drop of $\sim 50 \%$ ), the center line, and the southern limit of the shadow. The three solid lines indicate the same levels for the actual path of the shadow (derived from the analyses presented in this paper).

To address the issue of Pluto's changing atmospheric pressure and to again probe the structure of Pluto's atmosphere, we arranged for observations of the 2006 June 12 occultation of the star P384.2 (2UCAC 26039859: McDonald \& Elliot 2000; UCAC magnitude $=14.8$, where the UCAC bandpass is $579-642 \mathrm{~nm}$, between $V$ and $R$ : Zacharias et al. 2004). This occultation by Pluto was predicted to be visible from sites in southeastern Australia and most of New Zealand, and we selected a set that had moderate-aperture telescopes with good coverage of Pluto's shadow. Multiwavelength observations were not attempted because of the faintness of the star and lack of appropriate instrumentation. In this paper we describe the observations of this occultation (discussion of the prediction will be covered in a later publication). We also present a new method of analysis that allows for simultaneous modeling of the geometric solution for the occultation and Pluto's atmospheric structure. We combine the results from this occultation with those of past years $(1988,2002)$ to describe the structure of Pluto's atmosphere in 2006 and how it has changed over that time period. Our results are used to constrain Pluto's surface radius.

\section{OBSERVATIONS}

Observations of the 2006 June 12 Pluto occultation of the star P384.2 by our collaboration were successful at five sites (Fig. 1 and Table 1) in southeastern Australia: the $0.8 \mathrm{~m}$ telescope at the Star Castle Observatory in Black Springs (north of Adelaide), the $1 \mathrm{~m}$ telescope at the Mount Canopus Observatory near Hobart (University of Tasmania), the $1.8 \mathrm{~m}$ EOS (Electro Optic Systems) telescope at Mount Stromlo, the $2.3 \mathrm{~m}$ ANU (Australian National University) telescope at Siding Spring, and the $0.5 \mathrm{~m}$ Jubilee Telescope at Stockport Observatory (between Adelaide and Black Springs). The attempted observations at Mount John University Observatory in New Zealand were foiled by a massive regional power failure resulting from snowfall that broke a 60 year record. At the first four sites, we employed our frame-transfer cameras, POETS (Portable Occultation, Eclipse, and Transit System; 
TABLE 1

OBSERVATIONS

\begin{tabular}{|c|c|c|c|c|c|c|c|c|}
\hline Site & $\begin{array}{c}\text { Telescope } \\
\text { Diameter } \\
\text { (m) }\end{array}$ & East Longitude & Latitude & $\begin{array}{l}\text { Altitude } \\
(\mathrm{km})\end{array}$ & $\begin{array}{l}\text { Integration } \\
\text { Time } \\
\text { (s) }\end{array}$ & $\begin{array}{l}\text { Cycle } \\
\text { Time } \\
\text { (s) }\end{array}$ & $\mathrm{S} / \mathrm{N}^{\mathrm{a}}$ & Observers \\
\hline 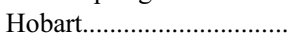 & 1.0 & 1472624 & -424818 & 0.290 & 0.498 & 0.500 & 33 & Souza, Jaskot, Giles, Greenhill \\
\hline Mount Stromlo..................... & 1.8 & 1490035 & -351857 & 0.813 & 0.198 & 0.200 & 29 & Gulbis, Kramer \\
\hline Siding Spring ....................... & 2.3 & 1490344 & -311617 & 1.205 & 0.198 & 0.200 & 96 & Adams, Lucas \\
\hline
\end{tabular}

Nоте.- Units of longitude and latitude are degrees, minutes, and seconds.

${ }^{a} \mathrm{The} \mathrm{S} / \mathrm{N}$ in the time that the shadow moves a distance of $60 \mathrm{~km}$ (approximately one pressure scale height), which was calculated from a portion of the light curves outside the occultation.

Souza et al. 2006), operating at $2 \mathrm{~Hz}$ at Black Springs and Hobart and $5 \mathrm{~Hz}$ at Mount Stromlo and Siding Spring. At Stockport, observations were made with a Meade DSI Pro CCD camera at an approximately $0.5 \mathrm{~Hz}$ cadence (each frame was time tagged), with exposure times of $\sim 1 \mathrm{~s}$. For POETS, the integration times were equal to the cycle times less the frame-transfer time of approximately $1.74 \mathrm{~ms}$ (for a typical vertical shift rate of $3.4 \mu \mathrm{s} \mathrm{per}$ row). While the CCD was being exposed with the next frame, we desired to read out the transfer region at the $1 \mathrm{MHz}$ rate in order to keep the read noise less than $6 e^{-}$pixel $^{-1}$, but the cadences used forced us either to bin the pixels $2 \times 2$ (as we did at Hobart) or to select a subframe (as we did at the other POETS stations) so that the readout could be completed prior to the next frame transfer initiated by the GPS clock.

In order to maximize the signal-to-noise ratio $(\mathrm{S} / \mathrm{N})$ of the data, no filters were employed. Hence, the observation passband was determined by the spectrum of the star, the quantum efficiency versus wavelength of the $\mathrm{CCD}$, and the transmission of the optics and atmosphere versus wavelength. We estimate the effective wavelength of this combination to be $\sim 7500 \AA$. A nearly full Moon only about $15^{\circ}$ away from Pluto posed some difficulties, especially at Mount Stromlo. Mount Stromlo was particularly affected because we installed our camera at the end of a laser ranging system that contained 15 separate optics that scattered the moonlight into the viewing field, increasing the noise from the background. These optics also reduced the throughput of starlight, decreasing the signal. The overall effect was to reduce the $\mathrm{S} / \mathrm{N}$ by a factor of about 2.6 below what would be expected for the size of the telescope.

Although the occultation by Pluto lasted just over a minute, data were recorded at the four sites with POETS cameras for approximately 80 minutes (centered on the occultation) to search for unknown satellites and debris in the system. The negative results of this search appear in an abstract (Pasachoff et al. 2006). Additional observations of this occultation were obtained by others, most notably at the $3.9 \mathrm{~m}$ Anglo-Australian Telescope, also located at Siding Spring Observatory (E. Young et al. 2007, in preparation).

\section{LIGHT-CURVE GENERATION}

In general, light curves were generated from raw data frames by summing the signal in a circular aperture centered on P384.2 (which also included Pluto and Charon), subtracting the background, and normalizing by field stars. There were some slight variations between analysis methods for each data set in order to optimize the $\mathrm{S} / \mathrm{N}$. In addition, the Hobart light curve was generated at Williams College, where slightly different procedures were used from those employed at MIT, where the other light curves were generated. First, because of intermittent light cloud cover at
Hobart and the effect of 15 optical elements in the beam at Mount Stromlo, these data sets were calibrated (bias frames subtracted and normalized by flat-field frames) prior to light-curve generation. Second, to correct for small transparency variations in the Earth's atmosphere, normalization by field stars included the mean signal from three stars for all stations except Hobart (six field stars) and Mount Stromlo (two field stars). To reduce the photon noise introduced by the field-star normalization procedure, each mean field-star signal (except Hobart, for which this was not a problem) was smoothed with a time-averaging function that had a width between 3 and 7 (time) integration bins (Table 1). Implicit in this procedure is the assumption that the transparency varies on timescales longer than those used to time-average the field-star signals.

Because Pluto-Charon is brighter than P384.2, centroiding algorithms will preferentially center on Pluto rather than on the occulted star near the time of occultation. Thus, we employed the method of centroiding a bright comparison star on each frame and applying a positional offset to accurately place the aperture used to generate the occultation light curves for all stations except Hobart. The positional offsets were calculated by identifying P 384.2 and three field stars on astrometric frames, when all objects were well separated prior to the event. These four stars were centroided, and their pixel positions were measured on more than 15 frames at each site. The average of the measured offsets between the brightest comparison star and P384.2 was applied to obtain the offset of the occultation light-curve aperture. The averages of the measured offsets between the comparison stars were applied to position the apertures used to generate field-star normalization data. The algorithm we employed to centroid the brightest comparison star evaluated a limited number of pixels surrounding the central position from the previous frame. Therefore, our technique was viable for only a small amount of telescope drifting. Image rotation at Mount Stromlo (an alt-az telescope) required that the offsets be functions of time. In this case, approximately 80 frames before and after the event were interpolated to generate the offsets. For Hobart, the same aperture was used on each frame used to generate the light curve.

We found that the light curves were sensitive to background calibration: selection of a background signal region located too far or in only one direction from P384.2 resulted in the unocculted stellar signal being sloped and/or having increased noise. This sensitivity is most likely due to the close proximity of the Moon, which created significant gradients in the background light. The best results for estimating background were obtained by selecting several regions from each image that bracketed the targets of interest (Pluto-Charon, P384.2, and the comparison stars). These regions were slightly different sizes and locations for each data set. The background signal regions were chosen to avoid background 
TABLE 2

Data Reduction Parameters

\begin{tabular}{|c|c|c|c|}
\hline Site & $\begin{array}{l}\text { Synthetic } \\
\text { Aperture }^{\mathrm{a}} \\
\text { (pixels) }\end{array}$ & $\begin{array}{l}\text { Synthetic } \\
\text { Aperture }^{\mathrm{a}} \\
(\operatorname{arcsec})\end{array}$ & $\begin{array}{c}\text { Nominal Background } \\
\text { Fraction }\left(f_{b}\right)^{\mathrm{b}}\end{array}$ \\
\hline Black Springs............... & 15 & 12.9 & 0.6796 \\
\hline 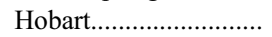 & $8^{\mathrm{b}}$ & 6.9 & 0.6898 \\
\hline Mount Stromlo............ & 8 & 4.4 & 0.6613 \\
\hline Siding Spring .............. & 21 & 8.4 & 0.6727 \\
\hline Stockport ....................... & 15 & 11 & 0.67 \\
\hline
\end{tabular}

${ }^{a}$ The shapes of all apertures were approximate circles, with the signal for pixels having their centers inside the aperture radius included.

b Data were binned $2 \times 2$, so this number represents a binned pixel.

stars, with the total area measuring at least 1.1 times the aperture area. The average value of the background regions was then subtracted from the summed signal in the light-curve aperture.

Light curves were generated over a range of aperture sizes, and the light curve with the highest $\mathrm{S} / \mathrm{N}$ at each site was selected for analysis. For all data sets, in all frames and all aperture sizes, the occultation light-curve aperture contained Pluto-Charon and P384.2. The optimal aperture diameters are given in Table 2, and the selected light curves are plotted in Figure 2 as functions of time.

In Figure 2, some light-curve features are immediately discernible. First, there is no obvious kink feature in any of the light curves: the flux does not show an abrupt change in slope during immersion or emersion, as it did in 1988. Next, the station closest to the center, Mount Stromlo, at a distance of about $150 \mathrm{~km}$, shows no evidence of a central flash (Elliot \& Olkin 1996) within the noise level of the data. Although a few small "spikes" are evident in the Siding Spring light curve, we see no large spikes during immersion or emersion. Note, however, the feature in the Siding Spring light curve just prior to emersion (small rise and subsequent dip) that indicates some atmospheric asymmetry. Finally, the light curves are somewhat rounded at the bottom and do not reach zero stellar flux. Thus, some starlight is detected throughout the occultation, having been refracted by Pluto's atmosphere but not completely attenuated. In $\S 6.3$.1 we give a detailed comparison between the Siding Spring light curve and data from previous years.

To illustrate the regions of Pluto's atmosphere probed by each light curve, we have plotted the trajectories of the apparent star position for each station in Figure 3. Because of refraction by Pluto's atmosphere, as the star approaches the limb the apparent radial motion slows considerably, but the tangential component continues. For every trajectory shown ("near limb"), there is a corresponding trajectory for light refracted from the opposite ("far") limb that is much weaker and may be totally cut off by the surface or extinction in the lower atmosphere. With these data we have no way of identifying the presence of a far-limb component (expected to be less than $1 \%$ of the unocculted star in any case).

\section{LIGHT-CURVE MODEL FITTING}

We analyzed the data by fitting a model with a power-law thermal structure (Elliot \& Young 1992) and assumed that the atmosphere has the same thermal structure globally above the 0.4 stellar flux level. Because the radiative time constants are so long, the atmospheric structure is expected to be the same around the body on theoretical grounds (Yelle \& Elliot 1997) and is borne out by model fits to the individual stations (the results of which agree

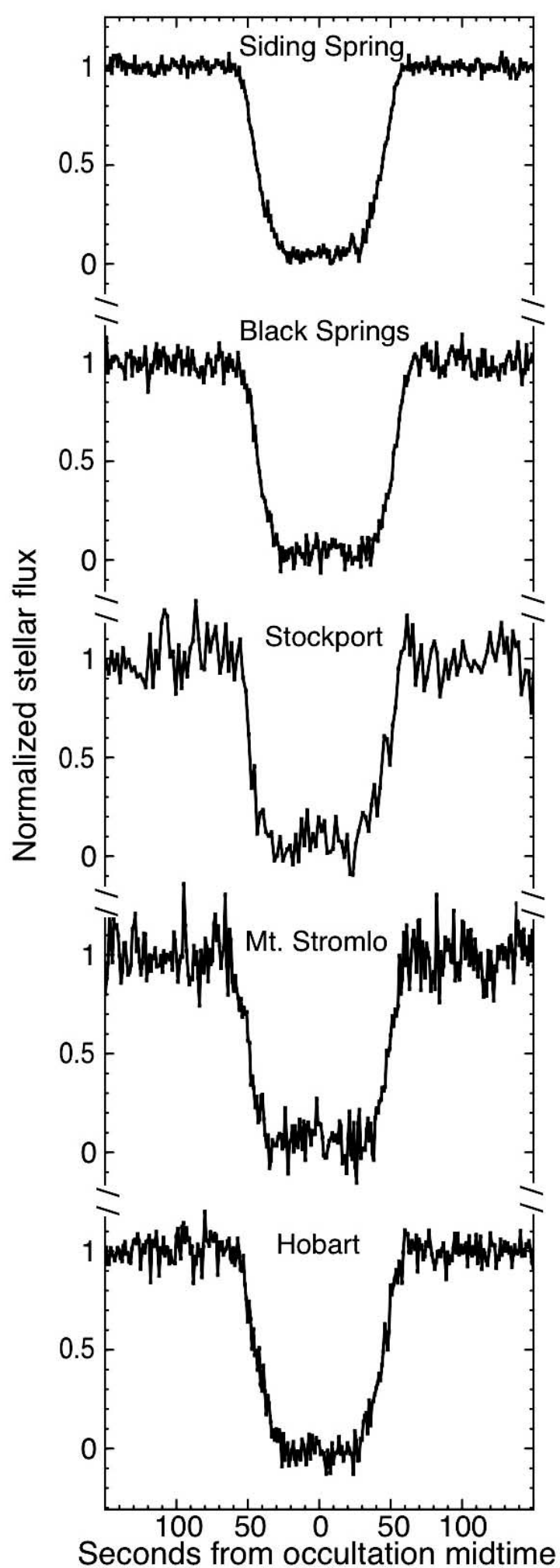

FIG. 2.- Observed light curves from each successful station. The light curves are plotted vs. time. They are averaged to an exposure time of $1 \mathrm{~s}$ and are displayed in order of decreasing station latitude. The prominent dip in each light curve is caused by the occultation by Pluto's atmosphere. The higher frequency variation is random noise, but we note a small feature caused by Pluto's atmosphere in the lower part of the Siding Spring curve, just prior to emersion. 


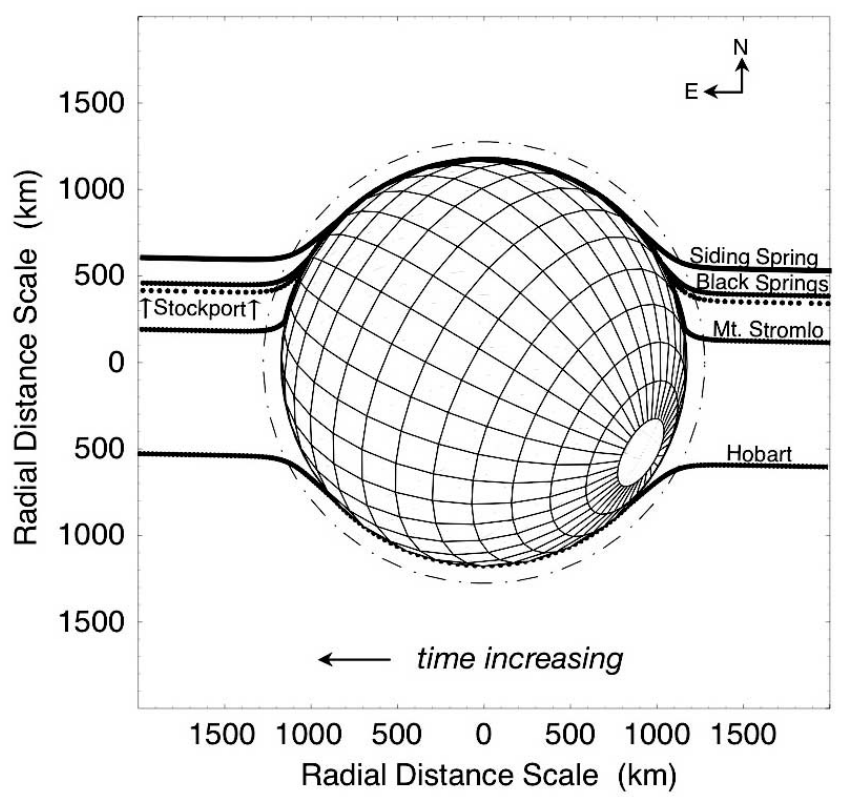

FIG. 3.- Paths of the stellar image around Pluto's limb during the occultation. These paths (in the plane of the sky) represent the locations at which the star would have been observed from each station if it were resolvable (an example of resolved images for a star occulted by Saturn is given by Nicholson et al. 1995). A small portion of the observed light may also have been contributed from the far limb, but that is not depicted here. These chord paths demonstrate the portions of Pluto's limb probed at each station and are plotted at the same time resolution as the light curves in Fig. 2. The dot-dashed line surrounding Pluto's figure marks the half-light radius, and Pluto's south pole (IAU convention) is in the lower right. The geocentric, sub-Earth point on Pluto at the time of the occultation was $-36.7^{\circ}$ latitude and $348.1^{\circ}$ longitude (IAU convention; suboccultation points on Pluto for each station are given in Table 7).

within their errors). The Elliot \& Young (1992) model postulates a thermal structure of the form $T(r)=T_{h}\left(r / r_{h}\right)^{b}$, where $T(r)$ is the temperature as a function of radius $(r), r_{h}$ is the half-light radius, $T_{h}$ is the temperature at half-light, and $b$ is a parameter describing the thermal gradient. For $b=0$, the atmosphere is isothermal. Since there is no abrupt drop in signal that would indicate an occultation by the limb of Pluto (Fig. 2), the surface radius was set to a value $(1105 \mathrm{~km})$ such that a surface occultation did not affect the model light curve. A one-limb model was used, and all five light curves were fit simultaneously.

The parameters of the fit are given in the first column of Table 3 . The global atmospheric parameters that applied to all light curves are (1) the half-light radius $\left(r_{h}\right)$, (2) index (b) the thermal-gradient, and (3) the equivalent isothermal gravitational to thermal energy ratio $\left(\lambda_{\text {iso }}\right)$ at the half-light radius. Note that $\lambda_{\text {iso }}=G M \mu / r_{h} k T_{h}-$ $5 b / 2$, where $G$ is the gravitational constant, $M$ is the mass of Pluto, $\mu$ is the mass of a gas molecule, and $k$ is Boltzmann's constant. See Elliot \& Young (1992) for an explanation of this equation and the details of how these quantities are used to calculate a model light curve.

The global astrometric parameters are the shadow center offsets in right ascension and declination, $f_{0}$ and $g_{0}$. As defined by Elliot et al. (1993), $f_{0}$ and $g_{0}$ refer to offsets from the difference between a reference position for the star and a reference ephemeris for Pluto. For the reference position of the star P384.2, we use the position, corrected for proper motion at the epoch of the occultation, taken from the UCAC2 catalog (Zacharias et al. 2004). This reference position is given in Table 4 . The source of our reference ephemeris for Pluto is the PLU013 light-time-corrected, geocentric ephemeris from the JPL (source files: PLU013, DE405; reference frame: ICRF/J2000.0). Three points from that ephem- eris are presented in Table 4. The coordinates in Table 1 were used with the procedure of Elliot et al. (1993) to calculate $f(t)$ and $g(t)$ as interpolation functions for each station, where $t$ is the time. The precision of these calculations was better than $0.1 \mathrm{~km}$, which required about three more digits of precision than given in Table 4. These extra digits were added as zeros.

Specific parameters that apply to each station individually are (1) the full-scale signal (when the star was not occulted), (2) the slope of the full-scale signal, and (3) the offset $\left(\Delta f_{b}\right)$ of the "background fraction" from its nominal value $\left(f_{b}\right.$, determined during the construction of the light curves). We call the background fraction the portion of the full-scale signal that was not attributable to the star. Since our light-curve-generating procedure should have removed the moonlight and bias levels, the background fraction should be only that fraction of the signal due to Pluto and Charon (i.e., $f_{b}=S_{\text {Pluto-Charon }} /\left[S_{\text {star }}+S_{\text {Pluto-Charon }}\right]$, where $S_{\text {Pluto-Charon }}$ is the full-scale signal from Pluto-Charon and $S_{\text {star }}$ is the full-scale signal from the star). Defined in this way, note that the background fraction has nothing to do with whatever residual stellar flux might be remaining during the middle of the occultation. The residual stellar flux for a given station depends on the properties of Pluto's atmosphere that control the shape of the light curve (i.e., thermal structure and extinction) and the minimum distance of the station from the center of Pluto's shadow.

Including the background-fraction offset as a potential fitted parameter allows us to test the consistency of four of our assumptions: (1) the process used for generating occultation light curves was valid (did not introduce systematic error), (2) the PlutoCharon background was accurately calibrated, (3) Pluto's atmosphere has circular symmetry in the plane perpendicular to the line of sight when the occultation was observed, and (4) the thermal structure of Pluto's atmosphere corresponding to light-curve flux levels above $\sim 0.4$ is globally the same. The test of these assumptions is possible because of the strong correlation among the parameters $\Delta f_{b}, \lambda_{\text {iso }}$, and $r_{h}$ in the model fits. Forcing $\lambda_{\text {iso }}$ and $r_{h}$ to be globally the same, but allowing $\Delta f_{b}$ to be free at one or more selected stations, allows the model to adjust $f_{b}$ at those stations to compensate for a violation of any of the assumptions just listed. A fitted value for $\Delta f_{b}$ consistent with 0.0 shows that the assumptions are self-consistent within the precision of the fit.

Potential single-station parameters also include an offset to the nominal time of the light curve and offsets to the $(f, g)$ coordinates. The former is useful in compensating for clock errors at individual stations, and the latter is used to compensate for any uncertainty in the station location. If the offsets to the $(f, g)$ coordinates are used for any particular station, then the $(f, g)$ coordinates for that station are updated with each iteration of the least-squares fit.

Sections covering a $360 \mathrm{~s}$ interval surrounding the occultation portion of each light curve were selected for fitting. To facilitate the weighting of the data points, each curve was (approximately) normalized between 0.0 (star fully occulted) and 1.0 (star fully visible). Weights used in the fitting were calculated from the variance of the pre-occultation signal. The nominal values of the background fraction for each station are given in Table 2. The background fractions for Black Springs, Hobart, Mount Stromlo, and Siding Spring were determined from frames taken when the images of Pluto-Charon and the star P384.2 were well separated. Since such frames were not available with the Stockport data set, we did not determine a background fraction for Stockport. The nominal value was set to 0.67 , which had no effect on the results, since an offset from this value was a free parameter in all model fits (Table 3). The background fractions would be expected to differ slightly among our stations (Table 2) because no filters 
TABLE 3

Model Fits to Light Curves

\begin{tabular}{|c|c|c|c|c|}
\hline Parameter & Fit 1 & Fit 2 & Fit 3 & Fit 4 \\
\hline 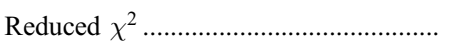 & 1.06922 & 1.06737 & 1.06441 & 1.06458 \\
\hline Half-light radius, $r_{h}(\mathrm{~km}) \ldots \ldots \ldots \ldots \ldots \ldots \ldots$ & $1279.2 \pm 3.2$ & $1276.1 \pm 3.5$ & $1273.4 \pm 8.7$ & $1282.5 \pm 4.0$ \\
\hline 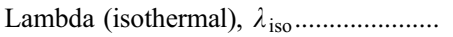 & $20.4 \pm 0.5$ & $18.3 \pm 0.8$ & $18.1 \pm 1.0$ & $19.7 \pm 1.1$ \\
\hline Thermal gradient power index, $b \ldots \ldots \ldots$ & 0.0 & $-2.2 \pm 0.7$ & $-2.2 \pm 0.7$ & $-1.8 \pm 0.9$ \\
\hline Offset in R.A., $f_{0}(\mathrm{~km}) \ldots \ldots \ldots \ldots \ldots \ldots \ldots \ldots$ & $-210.6 \pm 2.2$ & $-210.3 \pm 2.2$ & $-210.3 \pm 2.2$ & $-210.2 \pm 2.2$ \\
\hline 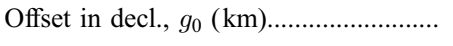 & $-1146.2 \pm 7.5$ & $-1145.7 \pm 7.7$ & $-1141.5 \pm 18.4$ & $-1141.2 \pm 9.4$ \\
\hline \multicolumn{5}{|c|}{ Black Springs } \\
\hline 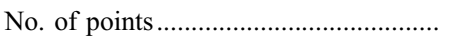 & 543 & 543 & 543 & 543 \\
\hline Background fraction offset, $\Delta f_{b} \ldots \ldots \ldots \ldots$ & 0.0 & 0.0 & $-0.020 \pm 0.010$ & 0.0 \\
\hline Slope & $0.15 \pm 0.23$ & $0.15 \pm 0.23$ & $0.15 \pm 0.23$ & $0.15 \pm 0.23$ \\
\hline 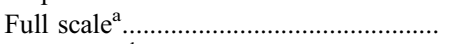 & $9967 \pm 28$ & $9961 \pm 28$ & $9972 \pm 29$ & $9970 \pm 29$ \\
\hline Time offset $^{\mathrm{b}}(\mathrm{s})$ & $4.42 \pm 0.22$ & $4.42 \pm 0.23$ & $4.41 \pm 0.22$ & $4.41 \pm 0.22$ \\
\hline \multicolumn{5}{|c|}{ Hobart } \\
\hline 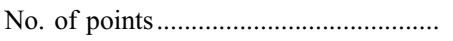 & 860 & 860 & 860 & 860 \\
\hline Background fraction offset, $\Delta f_{b} \ldots \ldots \ldots \ldots$ & 0.0 & 0.0 & $-0.014 \pm 0.028$ & 0.0 \\
\hline Slope & $0.33 \pm 0.18$ & $0.33 \pm 0.18$ & $0.33 \pm 0.18$ & $0.33 \pm 0.18$ \\
\hline 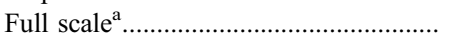 & $10,010 \pm 31$ & $10,006 \pm 31$ & $10,008 \pm 31$ & $10,008 \pm 31$ \\
\hline \multicolumn{5}{|c|}{ Mount Stromlo } \\
\hline 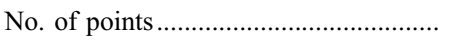 & 1324 & 1324 & 1324 & 1324 \\
\hline Background fraction offset, $\Delta f_{b} \ldots \ldots \ldots \ldots$ & 0.0 & 0.0 & $0.044 \pm 0.018$ & $0.058 \pm 0.016$ \\
\hline 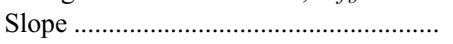 & $0.86 \pm 0.39$ & $0.86 \pm 0.39$ & $0.86 \pm 0.39$ & $0.86 \pm 0.39$ \\
\hline 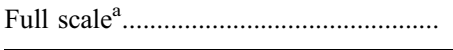 & $10,039 \pm 48$ & $10,033 \pm 48$ & $10,007 \pm 49$ & $10,007 \pm 49$ \\
\hline \multicolumn{5}{|c|}{ Siding Spring } \\
\hline 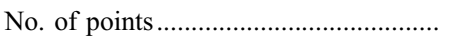 & 1387 & 1387 & 1387 & 1387 \\
\hline Background fraction offset, $\Delta f_{b} \ldots \ldots \ldots .$. & 0.0 & 0.0 & 0.0 & $0.015 \pm 0.009$ \\
\hline 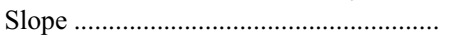 & $0.41 \pm 0.11$ & $0.41 \pm 0.11$ & $0.41 \pm 0.11$ & $0.41 \pm 0.11$ \\
\hline 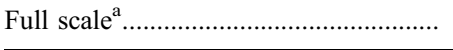 & $10,038 \pm 14$ & $10,032 \pm 14$ & $10,032 \pm 14$ & $10,032 \pm 14$ \\
\hline \multicolumn{5}{|c|}{ Stockport } \\
\hline 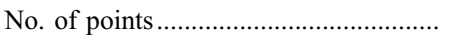 & 140 & 140 & 140 & 140 \\
\hline Background fraction offset, $\Delta f_{b} \ldots \ldots \ldots \ldots$ & $0.037 \pm 0.021$ & $0.032 \pm 0.021$ & $0.030 \pm 0.022$ & $0.043 \pm 0.021$ \\
\hline Slope & $0.0 \pm 0.8$ & $0.0 \pm 0.8$ & $0.0 \pm 0.8$ & $0.0 \pm 0.8$ \\
\hline 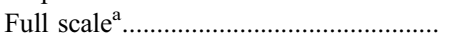 & $10,723 \pm 96$ & $10,720 \pm 96$ & $10,720 \pm 96$ & $10,720 \pm 96$ \\
\hline
\end{tabular}

were used, and (as was discussed in $\S 2$ ) the passband for each station was defined by the quantum efficiency versus wavelength response of the $\mathrm{CCD}$ and the combined transmission versus wavelength of the Earth's atmosphere and optical components of the individual systems. Hence, the background fractions for individ-

TABLE 4

Pluto and Star Positions

\begin{tabular}{cccc}
\hline \hline \multirow{2}{*}{ Body } & $\begin{array}{c}\text { Time } \\
\text { (UT on 2006 Jun 12) }\end{array}$ & R.A. (J2000.0) & Decl. (J2000.0) \\
\hline P384.2 ............................. & 120000 & 174112.0743 & -154134.575 \\
Pluto ...... & 150000 & 174112.4585 & -154134.529 \\
& 160000 & 174112.1887 & -154134.606 \\
& 170000 & 174111.9188 & -154134.684 \\
\hline
\end{tabular}

NotE.-Units of right ascension are hours, minutes, and seconds, and units of declination are degrees, arcminutes, and arcseconds. ual stations would be sensitive to color differences between PlutoCharon and the star.

The model fitting proceeded in two steps. First we fit all the light curves with the full model of Elliot \& Young (1992), including the extinction parameters, in order to get a model light curve that matched the data from each station reasonably well. Then we eliminated all points whose model fluxes were less than 0.4 . The flux cutoff of 0.4 was selected so that further fitting could include as much data as possible, but includes only points that correspond to the clear, nearly isothermal portion of the atmosphere (where there is no evidence of extinction or strong thermal gradients). As will be seen from the results of the fitting, this choice of cutoff flux level satisfied our criteria.

The second step was to simultaneously fit the model of Elliot \& Young (1992; with no extinction) to all light curves, using the selected data just described. Although many fits were carried out, we present just four in Table 3. Fit 1 is a forced isothermal solution with the background-fraction offsets fixed, except for 


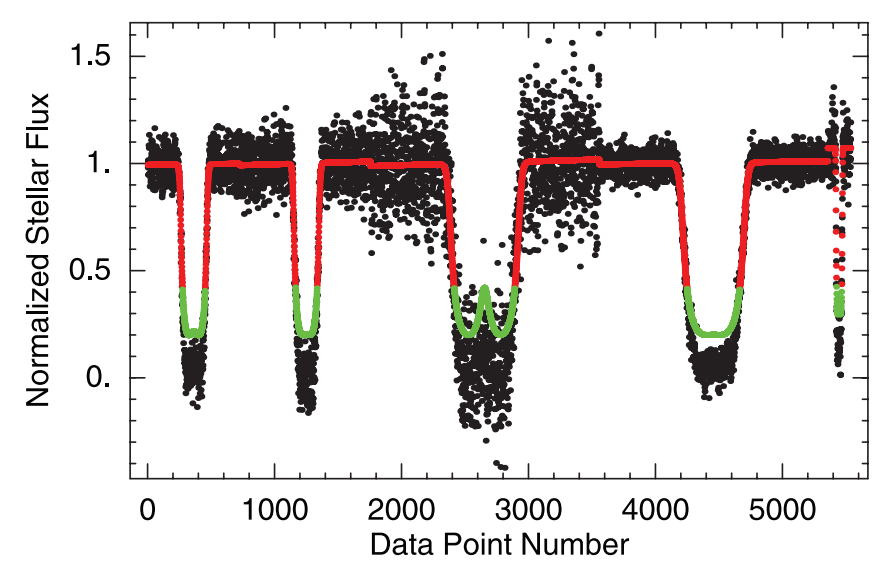

Fig. 4.- Model fits to light curves. The five light curves are plotted contiguously and indicated by the points. The order of the light curves is alphabetical: Black Springs, Hobart, Mount Stromlo, Siding Spring, and Stockport. Before merging, each light curve was normalized to a full scale close to 10,000 . The red line indicates the fitted global model (with parameters given by the second column in Table 3 in the region of the fit, which included only points that had corresponding model fluxes above 0.4 in units of normalized stellar flux; see text). Extension of the fitted model to fluxes below 0.4 is indicated by the green line segments. In all cases the green line segments lie well above the data, indicating that the model that fits the upper atmosphere does not apply to the lower atmosphere. Atmospheric extinction and/or a decrease in temperature with decreasing altitude would explain why the light curves go below the extrapolated model.

Stockport, whose background-fraction offset was allowed to vary in all fits. Fits $2-4$ allow the thermal-gradient parameter to be free. Fit 2 has the background offset parameters fixed. Fit 3 has only the background-fraction offset for Siding Spring fixed, and fit 4 has only the background-fraction parameter for Black Springs and Hobart fixed. We note that although the results vary somewhat more than the formal errors, most fits yield similar results, indicating that they are robust and do not depend strongly on any individual light curve.

Looking at the fit results, we note that the thermal-gradient parameter has a nearly $3 \sigma$ significance in fits $2-4$, so we reject the forced isothermal fit (fit 1). In fit 3, in which we let the background-fraction offsets for Black Springs, Hobart, and Mount Stromlo be free parameters, the fitted values for Black Springs and Hobart are 0.0 within 2.0 formal errors of the fit, while the value for the Mount Stromlo light curve disagrees with 0.0 by slightly more: 2.5 formal errors. In fit 4 , in which the background-fraction offset for Siding Spring is free, its fitted value agrees with 0.0 within 1.3 formal errors. Hence, the mutual consistency of the calibrated background fractions for our three best light curves leads us to believe that (1) we have calibrated them correctly, and (2) our key modeling assumptions are correct.

Therefore, although it does not have the lowest value for the reduced $\chi^{2}$, we have adopted the results of fit 2 because all the background-offset fractions (excluding Stockport) have been fixed at 0.0 , and it allows the thermal gradient in the upper atmosphere to be a free parameter. The results of this fit should give the best information from our data sets about Pluto's atmosphere.

The data and fitted model are plotted in Figure 4, where the data are indicated by the points and the fitted model (normalized flux greater than 0.4 ) is indicated by the red line. The green line shows the extension of the model into regions of unfitted data. In all cases the green line lies well above the data, indicating that the atmospheric structure for altitudes corresponding to a normalized stellar flux greater than $\sim 0.4$ does not extrapolate to lower altitudes. The data corresponding to the lower atmosphere lie below the model that fits the upper atmosphere, which is due to the onset of extinction and/or a drop in temperature at lower altitudes.

As can be seen in Figure 4, in spite of the high noise level of the Mount Stromlo data, that station's proximity to the center of the shadow would have produced a detectable central flash had the same atmospheric structure extended to lower altitudes in the atmosphere. From the fitted parameters (Table 3) we have derived several additional parameters of interest from the results of our adopted fit 2, which are given in Table 5. The errors are propagated from the formal errors and correlation coefficients of the fitted parameters. The temperature at the half-light radius of $97 \pm 5 \mathrm{~K}$ agrees with that found in previous years (see $\S 6.3$ ) and is consistent with the atmosphere probed by the occultation being controlled by radiative equilibrium with methane (Strobel et al. 1996), a small amount of which has been detected by Young et al. (1997).

The model fit also yields a geometric solution for the occultation through the fitted parameters $f_{0}$ and $g_{0}$. These parameters give the offsets in the shadow plane relative to a set of reference coordinates for the star and the occulting body (see Table 4 and Elliot et al. 1993). In Table 6 we compare the results of the astrometric solution with the last MIT prediction prior to the event. ${ }^{13}$ Links to predictions by other groups are also given at the MIT prediction Web site. The MIT prediction was in error by $0.010^{\prime \prime}$ ( 0.18 Pluto shadow radii) in the location of the occultation path and had an error of 02:23 in the predicted time (2.79 Pluto shadow radii). Thus, the MIT prediction was acceptably accurate in the location of the path, but grossly wrong in the direction along the path. We shall assess these errors in a future publication about our prediction methods. The geometric solution has also been used to establish accurate suboccultation coordinates on Pluto at the points of half-light for each station listed in Table 7.

\section{INVERSION}

Under the assumption of a clear atmosphere, we have used our inversion technique (Elliot et al. 2003a) to find the thermal

\footnotetext{
${ }^{13}$ See http://occult.mit.edu/research/occultations/Pluto/P384.2-preds/P384.2AST-1.8.html
}

TABLE 5

Quantities Derived from Fitted Parameters

\begin{tabular}{|c|c|c|c|c|}
\hline Parameter & Fit 1 & Fit 2 & Fit 3 & Fit 4 \\
\hline Shadow radius for half-light $(\mathrm{km})$ & $1211 \pm 4$ & $1208 \pm 4$ & $1205 \pm 10$ & $1218 \pm 5$ \\
\hline Pressure scale height $(\mathrm{km})$ & $63.1 \pm 1.5$ & $53.8 \pm 2.9$ & $54.0 \pm 2.9$ & $53.5 \pm 3.3$ \\
\hline 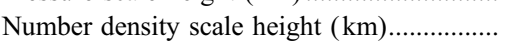 & $63.1 \pm 1.5$ & $59.3 \pm 1.8$ & $59.6 \pm 1.9$ & $57.7 \pm 2.1$ \\
\hline Pressure ( $\mu$ bar) & $1.94 \pm 0.12$ & $1.58 \pm 0.14$ & $1.61 \pm 0.16$ & $1.47 \pm 0.15$ \\
\hline 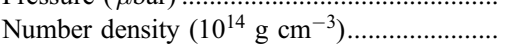 & $1.24 \pm 0.05$ & $1.18 \pm 0.05$ & $1.20 \pm 0.06$ & $1.11 \pm 0.06$ \\
\hline Temperature $(\mathrm{K})$ & $113 \pm 3$ & $97 \pm 5$ & $98 \pm 5$ & $95 \pm 6$ \\
\hline Temperature gradient $\left(\mathrm{K} \mathrm{km}^{-1}\right) \ldots \ldots \ldots \ldots \ldots \ldots \ldots$ & 0.0 & $-0.17 \pm 0.05$ & $-0.17 \pm 0.05$ & $-0.13 \pm 0.06$ \\
\hline
\end{tabular}

NotE.-At the half-light radius. 
TABLE 6

Comparison of the Astrometric Solution with the Mit Occultation Prediction

\begin{tabular}{|c|c|c|}
\hline Parameter & Geocentric Closest Approach ${ }^{\mathrm{a}}$ & Geocentric Midtime (UT on 2007 Jun 12) \\
\hline 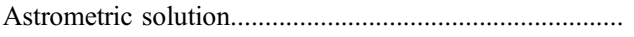 & $2537 \mathrm{~km}$ & $16: 25: 15$ \\
\hline Predicted solution & $2316 \mathrm{~km}$ & $16: 22: 52$ \\
\hline 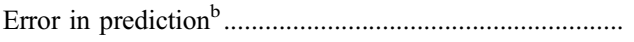 & $221 \mathrm{~km}$ & $00: 02: 23$ \\
\hline Error in prediction & $0.010^{\prime \prime}$ & $0.154^{\prime \prime}$ \\
\hline Error in prediction (Pluto shadow radii)............................. & 0.18 & 2.79 \\
\hline
\end{tabular}

a These distances are south of the geocenter.

b The prediction was $221 \mathrm{~km}$ north and 2:23 earlier than indicated by the astrometric solution.

structure. Using boundary conditions from the adopted model fit (No. 2, given in Table 3, which applies to those portions of the light curve with fluxes above 0.4), inversion was performed on the remainder of the Siding Spring light curve (fluxes below 0.4 ). The inversion parameters are listed in Table 8 , and the thermal profiles corresponding to the immersion and emersion portions of the light curve are shown in Figure 5. The upper part of the inverted profiles is dominated by the boundary conditions, showing the negative thermal gradient from the model fit. The errors in neighboring points are highly correlated, so error bars are indicative of the error in the absolute temperature, rather than the relative error of the individual points. At lower altitudes, the profiles show a strong gradient to lower temperatures, as required to reach the surface-ice temperature of $\sim 40 \mathrm{~K}$. The differences between the two profiles for radii below $\sim 1205 \mathrm{~km}$ are significant and are the result of asymmetry in the Siding Spring light curve.

\section{DISCUSSION}

\subsection{Comparison with a Physical Model}

In Figure 5 we have also plotted one of the thermal models published by Strobel et al. (1996), which is a full radiative-conductive model that assumes hydrostatic equilibrium and includes (1) solar radiation, (2) thermal conduction, (3) heating by the 2.3 and $3.3 \mu \mathrm{m}$ bands of $\mathrm{CH}_{4}$, (4) cooling by the $7.6 \mu \mathrm{m} \mathrm{CH}_{4}$ band, and (5) cooling by $\mathrm{CO}$ rotational line emission. The case selected for Figure 5 has a gradient in the $\mathrm{CH}_{4}$ mixing ratio of the functional form $\gamma(n)=\gamma_{0}\left(n / n_{0}\right)^{\beta}$, where $n$ is the number density, $\gamma_{0}$ is the surface $\mathrm{CH}_{4}$ mixing ratio of $3.6 \%, n_{0}$ is the surface number density, and the power index $\beta=1.5$ (see Fig. 11 in Strobel et al. 1996). Other than adjusting the radius scale to provide the best (visually estimated) fit to our inversion results, no attempt was made to optimize the parameters of the Strobel et al. (1996) model. This figure is meant to demonstrate that a model that includes only the physical effects taken into account by Strobel et al. (1996) can qualitatively match our inversion results.

The decrease in temperature with altitude at the top of the profile is due to CO cooling, and below this, the shape of the profile depends on the amount of $\mathrm{CH}_{4}$ and how it is mixed. The model curve reaches the expected surface-ice temperature at approximately $1184 \mathrm{~km}$. In the selected model, the total amount of $\mathrm{CH}_{4}$ is in agreement with the measurement of Young et al. (1997). It is fortuitous, perhaps, that one of the cases published by Strobel et al. (1996) matches our results so well. Fine-tuning the model parameters would result in an even better fit.

Of course, one can also obtain a temperature profile that matches the $1988 \mathrm{KAO}$ data by inversion (Elliot et al. 2003a), under the assumption of no extinction. The resulting temperature profiles have thermal gradients as high as $10 \mathrm{~K} \mathrm{~km}^{-1}$ at a radius of $1200 \mathrm{~km}$. Such large gradients can only marginally be produced in the physical model of Strobel et al. (1996) in a thermal profile that matches the entire light curve. We are aware of no published physical model for Pluto's atmosphere that has been shown to reproduce the $1988 \mathrm{KAO}$ light curve. Even more extreme thermal gradients $\left(30 \mathrm{~K} \mathrm{~km}^{-1}\right)$ were discussed by Stansberry et al. (1994) in the context of matching the KAO light curve by adjusting the parameters of a Bates temperature profile. However, the Bates profile is a mathematical convenience and not a physical model.

Turning our attention to extinction models, in their initial analysis of the $1988 \mathrm{KAO}$ light curve, Elliot et al. (1989) considered that the kink was due to extinction that was abruptly turning on at the radius in the atmosphere that corresponded to the location of the kink in the light curve. Below the turn-on radius, the extinction exponentially increases with decreasing radius, but follows a scale height that is about a factor of 2 less than that followed by the atmospheric gases. As refined by Elliot \& Young (1992), this model describes the extinction in the atmosphere by three parameters: the turn-on radius, scale height, and the radius at which the slant-path optical depth (along the path followed by the occulted starlight) equals 1.0. The physical basis for extinction was investigated by Stansberry et al. (1989), who considered steady state models for haze production and sedimentation. They found that the optical depth would not be sufficient to produce the extinction levels required to explain the $1988 \mathrm{KAO}$ light curve and that the upper boundary of the haze would be diffuse, not sharp.

Hence, the extinction and thermal-gradient models have achieved the same limited degree of success in modeling the $1988 \mathrm{KAO}$

TABLE 7

Suboccultation Coordinates on Pluto

\begin{tabular}{|c|c|c|c|c|}
\hline Site & $\begin{array}{l}\text { Immersion Longitude } \\
\text { (deg) }\end{array}$ & $\begin{array}{l}\text { Immersion Latitude } \\
\text { (deg) }\end{array}$ & $\begin{array}{l}\text { Emersion Longitude } \\
\text { (deg) }\end{array}$ & $\begin{array}{c}\text { Emersion Latitude } \\
\text { (deg) }\end{array}$ \\
\hline 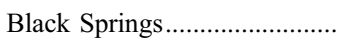 & 119.6 & -35.4 & 352.8 & 53.3 \\
\hline 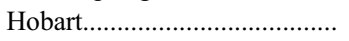 & 184.8 & -53.2 & 293.8 & 30.9 \\
\hline 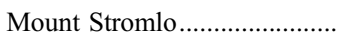 & 132.7 & -43.6 & 332.1 & 50.5 \\
\hline 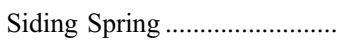 & 113.1 & -30.0 & 5.2 & 53.2 \\
\hline 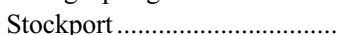 & 121.7 & -36.9 & 350.2 & 53.2 \\
\hline
\end{tabular}

NoтE.-Coordinates are expressed using the IAU convention, at the half-light radius. 
TABLE 8

INVERSION PARAMETERS

\begin{tabular}{|c|c|}
\hline Parameter & Value \\
\hline Distance to Pluto $\left(10^{9} \mathrm{~km}\right)$ & 4.506 \\
\hline Pluto mass $\left(10^{22} \mathrm{~kg}\right)$ & 1.305 \\
\hline 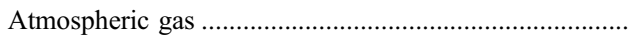 & $\mathrm{N}_{2}$ \\
\hline Mean molecular weight $(\mathrm{amu})$ & 28.01 \\
\hline Refractivity at STP $\left(10^{-4}\right)$ & 2.82 \\
\hline Integration upper limit ............................... & $\infty$ \\
\hline Series order & 2 \\
\hline Radial resolution, $\Delta y(\mathrm{~km})$ & $3.9-63.1$ \\
\hline Shell thickness, $\Delta r(\mathrm{~km})$ & $1.0-2.2$ \\
\hline 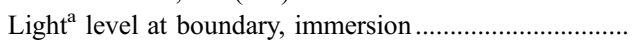 & 0.448 \\
\hline 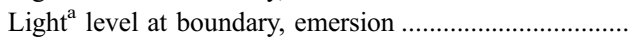 & 0.398 \\
\hline
\end{tabular}

Note.-For definitions and a discussion of the effect of the various inversion parameters on the results, see Elliot et al. (2003a).

${ }^{\text {a }}$ Normalized starlight.

light curve: the thermal gradients or levels of extinction needed to match the light curve are more severe than can be produced by current physical models. To our knowledge, models that combine thermal gradients and extinction have not been explored. One consequence of this uncertainty about whether to apply a thermal gradient or extinction model to the light curve is that the radius scale in the atmosphere depends on the choice of model, leaving us with a further uncertainty in the radius scale in Pluto's atmosphere below $\sim 1210 \mathrm{~km}$.

\subsection{Pluto's Surface Radius}

Extrapolating the thermal gradients from the inversion temperature profile to their intersection with the vapor-pressure equilibrium profile (Brown \& Ziegler 1980) for $\mathrm{N}_{2}$ indicates a surface radius of $1168 \pm 10 \mathrm{~km}$ (see Fig. 5 and Table 9). This implies a density of $1.96 \pm 0.05 \mathrm{~g} \mathrm{~cm}^{-3}$, greater than that of Pluto's moon Charon (Gulbis et al. 2006; Person et al. 2006), but virtually the same as Neptune's moon Triton (McKinnon et al. 1995).

We emphasize that an assumption of the inversion method is a clear atmosphere. If any extinction is present, the temperature gradients needed to explain the data will not be so great. Indeed, the evidence for extinction from the multiwavelength observations made in 2002 (Elliot et al. 2003b) would suggest that although the light curve in 2006 has greater flux along the bottom than in 2002, perhaps in 2006 some amount of extinction was still present. If extinction is present in the 2006 light curve, the inversion temperature profiles in Figure 5 would indicate lower temperatures than Pluto's atmospheric temperatures by an amount that depends

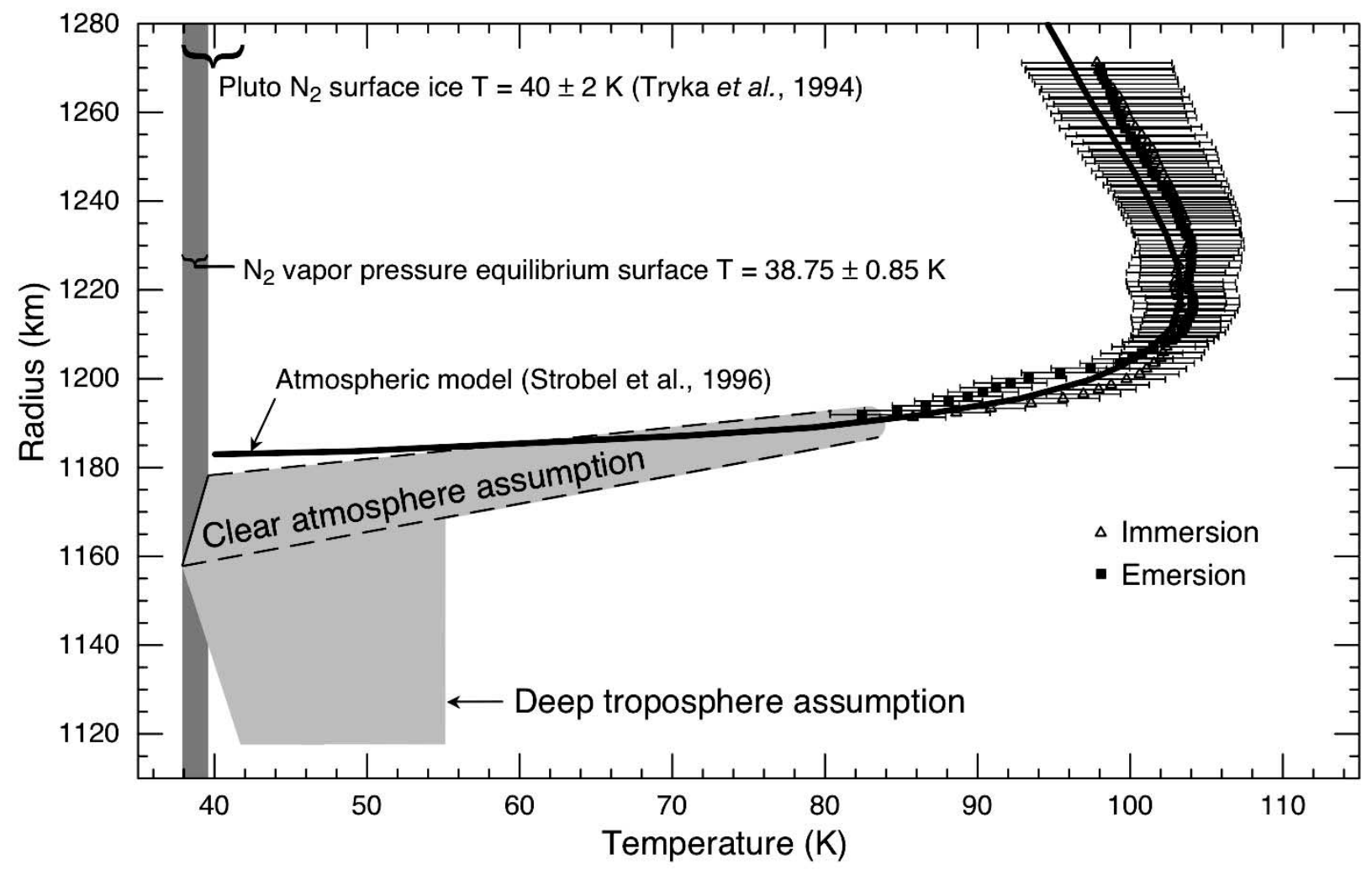

FIG. 5. - Pluto's atmospheric temperature profile for 2006. Temperatures obtained from inversion (Elliot et al. 2003a) of the Siding Spring light curve are plotted vs. distance from the center of Pluto. Immersion data points are represented by triangles, and emersion data points are squares. Boundary conditions for the half-light radius $\left(r_{h}\right)$, the thermalgradient index $(b)$, and the equivalent isothermal gravitational-to-thermal-energy ratio $\left(\lambda_{\text {iso }}\right)$ are fixed from model fit 2 (see Table 3 ). The asymmetry between immersion and emersion in the lower atmosphere, seen in the light curves, is also apparent here. The inversion results are well matched by an atmospheric model from Strobel et al. (1996), discussed in $\S 6.1$ and plotted as the solid line. A surface radius of $1183 \mathrm{~km}$ has been used for the atmospheric model to align it with the data. The negative temperature gradient at higher altitudes is a result of $\mathrm{CO}$ cooling. While the atmospheric structure below the inversion region is unknown, it is instructive to assume that the temperature continues to follow the gradient of the lowest altitude data points. A linear temperature extrapolation below $1197 \mathrm{~km}$, combined with the temperature differential between the final immersion and emersion data points, returns a $3 \sigma$ temperature gradient range of $2.2 \pm 0.6 \mathrm{~K} \mathrm{~km}^{-1}$ (shown as the light gray region in the plot). Assuming hydrostatic equilibrium, the extrapolated temperature profiles are applied to calculate pressure profiles. The temperature range at which the extrapolated pressures are consistent with the vapor-pressure equilibrium profile for $\mathrm{N}_{2}$ surface ice (Brown \& Ziegler 1980) is represented by the dark gray region and is consistent with measurements of Pluto's surface-ice temperature (Tryka et al. 1994; Gurwell $\&$ Butler 2005). The extrapolated temperature profile indicates a surface radius of $1168 \pm 10 \mathrm{~km}$. If extinction is present, the actual thermal gradient would be weaker, resulting in a smaller inferred surface radius. The light gray region extending below the temperature extrapolation represents the parameter space for a possible troposphere extending up to $48 \mathrm{~km}$ and having lapse rates ranging from wet pseudo-adiabat to dry adiabat (following Stansberry et al. 1994). 
TABLE 9

Scenarios for Pluto's Lower Atmosphere

\begin{tabular}{|c|c|c|}
\hline Parameter & No Troposphere ${ }^{\mathrm{a}}$ & $\begin{array}{l}\text { Troposphere } \\
\text { (48 km thick) }\end{array}$ \\
\hline Surface radius $(\mathrm{km})$ & $1168 \pm 10$ & $\sim 1120$ \\
\hline 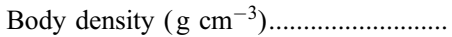 & $1.96 \pm 0.05$ & $\sim 2.2$ \\
\hline 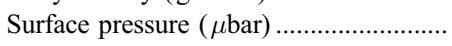 & $30_{-12}^{+17}$ & $\sim 310$ \\
\hline $\mathrm{N}_{2}$ surface-ice temperature $(\mathrm{K})$............. & $38.75 \pm 0.85$ & $\sim 43$ \\
\hline
\end{tabular}

${ }^{\text {a }}$ Based on extrapolation of the temperature profiles of Fig. 5 (derived under the assumptions of a clear atmosphere in hydrostatic equilibrium).

on the extinction. Hence, in the presence of extinction, the extrapolation of the temperature inversion profiles yields only an upper limit on Pluto's surface radius of $1178 \mathrm{~km}$. The Strobel et al. (1996) model plotted in Figure 5 that closely matches our inversion results would give an upper limit of $1184 \mathrm{~km}$ on the surface radius.

A third possibility is the deep troposphere scenario proposed by Stansberry et al. (1994), who considered models for tropospheric depths ranging between 10 and $40 \mathrm{~km}$. Displayed in Figure 5 is an approximate deep troposphere, constructed by adding a $48 \mathrm{~km}$ section below the atmospheric structure that we just considered. This is a crude approximation, and we have not compared the occultation light curves implied by these temperature profiles with our data. For comparison, the deep troposphere approximation provides a radius, density, surface pressure, and the corresponding $\mathrm{N}_{2}$ ice temperature listed in Table 9. The presence of a troposphere would allow the surface radius of Pluto implied by the stellar occultation data to be less than the value of $1168 \pm 10 \mathrm{~km}$ for no troposphere. As an example, for a $\sim 48 \mathrm{~km}$ troposphere, the surface radius would be $1120 \mathrm{~km}$ and the bulk density of Pluto would be $2.2 \mathrm{~g} \mathrm{~cm}^{-3}$, about $10 \%$ greater than that for Triton (McKinnon et al. 1995). In support of a troposphere on Pluto, we note that Triton - a body of similar size, atmospheric composition, density, and current solar distance - has a $\sim 50 \mathrm{~km}$ troposphere (Elliot et al. 2000).

Accounting for all possibilities discussed above, we can constrain the range for Pluto's surface radius to be between 1120 and $1184 \mathrm{~km}$ (see Fig. 5; the lower limit extends even lower if Pluto has a troposphere thicker than $48 \mathrm{~km}$ ). This range of allowed surface radii can be compared with the surface radii derived from the mutual events that occurred in 1985-1989 (Binzel \& Hubbard 1997; Tholen \& Buie 1997). First we note that mutualevent observations record reflected light, and hence the data analysis should account for an unknown amount of limb darkening (or brightening). If hazes or clouds are present, one would expect these to be thickest at the limbs, where they would contribute most to the reflected light, but in a manner that might not be consistent with the limb-darkening (or brightening) law followed by the rest of Pluto's disk.

With these caveats, values for Pluto's surface radius were $1151 \pm$ $6 \mathrm{~km}$ by Tholen \& Buie (1990) and $1151 \pm 4 \mathrm{~km}$ by Reinsch et al. (1994). Neither of these analyses included limb darkening (and assumed a uniformly bright disk), so they should be considered as lower limits. On the other hand, Young \& Binzel (1994) included limb darkening in their analysis and found $1173 \pm 23 \mathrm{~km}$ for Pluto's surface radius.

Considering these results, we could use $\sim 1145 \mathrm{~km}$ as the lower limit on Pluto's surface radius allowed by the mutual events, if it were not for the fact that none of the analyses accounted for the possibility of light reflected by haze or clouds at the limb. If these effects are large, then the surface radius could be signifi- cantly less than $1145 \mathrm{~km}$. In summary, we feel that $1120-1184 \mathrm{~km}$ $(1152 \pm 32 \mathrm{~km})$-allowed by a combination of the stellar occultation data and constraints imposed by models for the atmospheric structure - represents the current best estimate for Pluto's surface radius.

\subsection{Comparison of Atmospheric Structure: 1988-2006}

\subsubsection{Changing Extinction?}

Our light curve with the greatest $\mathrm{S} / \mathrm{N}$ is that obtained with the $2.3 \mathrm{~m}$ ANU telescope at Siding Spring (see Fig. 2). The S/N of these data is comparable to that of the best light curves obtained for Pluto occultations in 1988 (KAO, Elliot et al. 1989) and 2002 (University of Hawaii $2.2 \mathrm{~m}$, Pasachoff et al. 2005). It is instructive to put all three light curves on a common scale, as displayed in Figure 6, where we show the normalized flux versus distance from the center of Pluto's shadow on Earth for each year. Progressing through the years, we see that just below the half-light level, the 1988 curve drops abruptly and has a flat bottom where the starlight has been totally extinguished. The 2002 light curve does not drop as abruptly, but eventually has a flat bottom, while the light curve from 2006 has a bowl-shaped bottom, indicating that the flux from the star never reached zero.

The main drop in the light curves is caused by the exponential relationship between atmospheric number density and radius. A faster drop - as most strongly exhibited by the 1988 light curve below half-light - may be caused by the onset of extinction and/or by a thermal gradient. Both explanations have been explored for the 1988 data (Elliot et al. 1989; Stansberry et al. 1989; Elliot \& Young 1992; Eshleman 1989; Hubbard et al. 1990). If the atmosphere is globally homogeneous and the thermal structure has been the same, one way to explain the progression of light-curve shapes over the years is that significant extinction was introduced into Pluto's atmosphere prior to the 1988 observations, and since then the atmosphere has been clearing up (on a timescale of years). Since the settling timescales for particles in the atmosphere is so short ( $\sim 1$ day for $10 \mu \mathrm{m}$ aerosols, in the absence of winds), the timescale of the clearing reflects the timescale of the process causing the extinction. Steady state haze production by processes known to be operating on Titan, Uranus, and Neptune does not produce an adequate amount of extinction (Stansberry et al. 1989; Krasnopolsky \& Cruikshank 1999) to explain the 1988 KAO light curve. The plumes on Triton (Soderblom et al. 1990) give us an example of injected extinction that has a sharp upper boundary, although the plumes are sparse and of very limited spatial extent - certainly not the global extinction that we would require for Pluto. Perhaps a more extreme example of Triton's plumes occurred on Pluto.

\subsubsection{Thermal Structure}

In order to compare the thermal structure of the upper atmosphere in 1988, 2002, and 2006, we present the atmospheric parameters derived from the fit of the Elliot \& Young (1992) model in Table 10. For 1988 and 2002, these values are derived from model fits to all the light curves (M. J. Person et al. 2007, in preparation), in the same manner as we analyzed the 2006 data. Uncertainties in parameters are their formal errors from the fits. In addition, as discussed before in the context of the 2006 data, systematic errors can be introduced in the process of (1) generating light curves from the individual CCD frames, (2) determining the fraction of the total signal attributable to background light, and (3) modeling the atmospheric structure. The magnitudes of any systematic errors are difficult to determine precisely, but may be as large as twice the formal errors. 

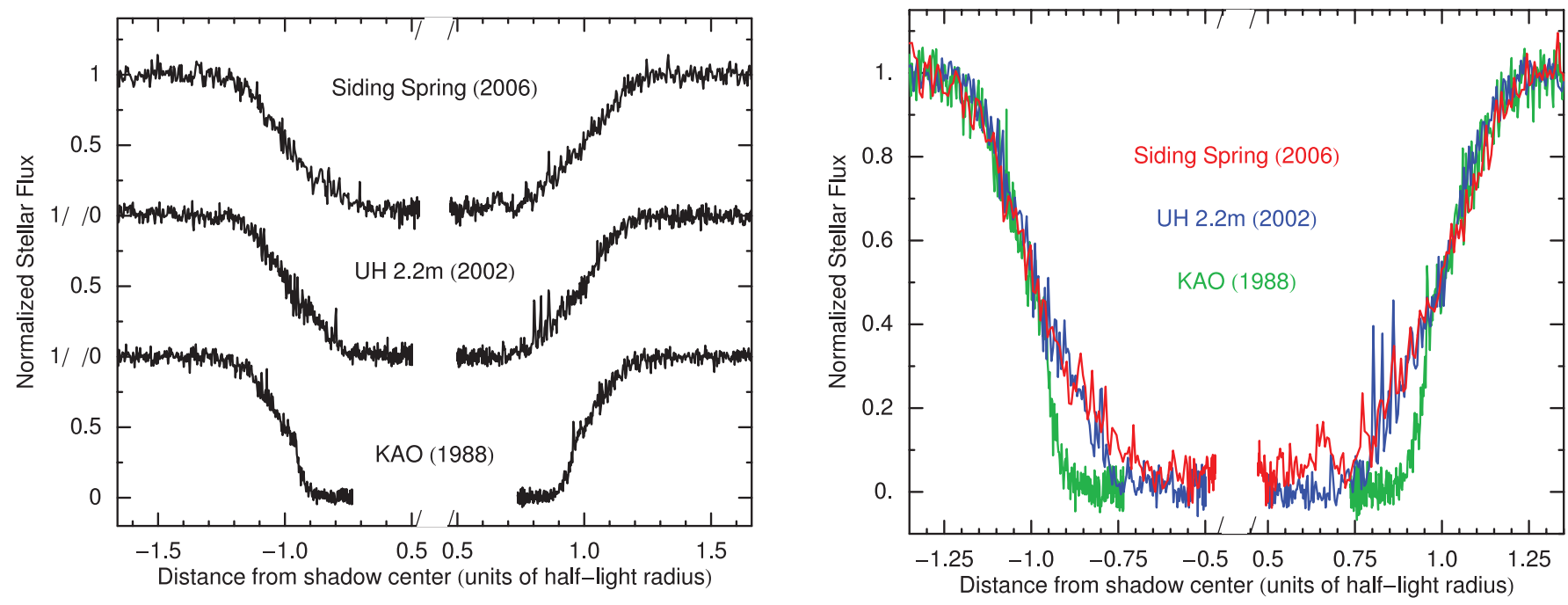

FIG. 6.-Comparison of Pluto occultation light curves in 1988 (0.9 m telescope on NASA's KAO), 2002 (University of Hawaii $2.2 \mathrm{~m}$ telescope), and 2006 (2.3 m telescope at Siding Spring). Left: Observed flux for each event displayed vs. distance from the shadow center. Note that this scale is broken in the middle, since none of the stations followed a path that intersected the shadow center, and that the light curves for 1988 and 2002 stop short of the minimum distance achieved at Siding Spring in 2006. These data are plotted at full time resolution, $2 \mathrm{~Hz}$ in 2002 and $5 \mathrm{~Hz}$ in 1988 and 2006. Right: Each of the three light curves plotted in a different color, with all three overlaid, to allow direct comparison of profiles. For clarity, the 2002 and 2006 data are binned by 2 over time. The evolution of the light-curve profile from a steep drop in 1988 to a more rounded baseline in 2006 is evident. Spikes are visible in the lower part of Pluto's atmosphere in 2002 and, on a smaller scale, in 1988 and 2006 . In addition, the 2006 light curve is asymmetric, with a significant feature just prior to emersion.

The most obvious change is the large increase in half-light radius in Pluto's atmosphere between 1988 and 2002. Stated another way, the pressure at a given radius had approximately doubled between 1988 and 2002. Note that the half-light radius in Pluto's atmosphere is approximately one pressure scale height greater than the half-light shadow radius (discussed in $\S$ 6.3.3). Differences in the pressure scale height, temperature, and thermal gradient are not statistically significant: there is no evidence for seasonal change in the thermal structure of the upper atmosphere (above $\sim 1210 \mathrm{~km}$ ) in the 1988-2006 time period.

\subsubsection{Half-Light Shadow Radius and Surface Pressure}

Finally, we address the question of the large pressure change in Pluto's atmosphere, which was noted between 1988 and 2002 (Elliot et al. 2003b; Sicardy et al. 2003). An accurately determined quantity from occultation data that is related to the surface pressure is the half-light radius as observed on Earth in the occultation shadow, since this quantity does not depend on any model of Pluto's atmosphere. The shadow radius is derived under the assumption that Pluto's atmosphere is spherically symmetric. Strictly speaking, the radius at which the light level drops to 0.5 depends on integrating the second derivative of the refractivity along the atmospheric path taken by the starlight (see eq. [2.7] and the immediately preceding equations in Elliot \& Young 1992). Assuming the atmospheric composition is homogeneous in that part of the atmosphere, the refractivity will be proportional to the number density, and we would be integrating the second derivative of the number density. If the atmospheric structure above the half-light level were the same over the time period of comparison (see Fig. 6 and Table 10 for evidence that it was), then the half-light radius will be a robust indicator of any change in the atmospheric structure below.

The quantity most directly affecting the value of the half-light radius will be the surface pressure, although a change in the thermal structure (without a change of surface pressure) could change the half-light radius as well. We have considered other approaches to describing the pressure changes, such as the radius of a given pressure within the atmosphere (e.g., Table 10), but such derived quantities are less connected with what is directly measured (the light curve), and hence they are subject to larger random error bars and further uncertainty inherent in assumptions about Pluto's atmosphere needed to derive them.

We have plotted values for the shadow half-light radius in Figure 7, where the error bars on the half-light radii in the shadow include all sources of error, as discussed below. Also plotted in Figure 7 is a frost-transport model from Hansen \& Paige (1996,

TABLE 10

Pluto's Upper Atmosphere (1988-2006)

\begin{tabular}{|c|c|c|c|}
\hline Parameter & 1988 Jun $9^{\text {a }}$ & 2002 Aug $21^{\mathrm{a}}$ & 2006 Jun 12 \\
\hline 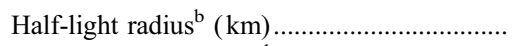 & $1233 \pm 4$ & $1279 \pm 5$ & $1276 \pm 4$ \\
\hline 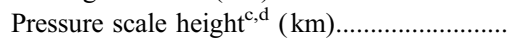 & $56 \pm 5$ & $61 \pm 4$ & $54 \pm 3$ \\
\hline Temperature $^{\mathrm{c}, \mathrm{d}, \mathrm{e}}(\mathrm{K})$ & $114 \pm 10$ & $108 \pm 9$ & $97 \pm 5$ \\
\hline Temperature gradient ${ }^{\mathrm{c}, \mathrm{d}}\left(\mathrm{K} \mathrm{km}^{-1}\right) \ldots \ldots \ldots \ldots \ldots \ldots$ & $-0.29 \pm 0.56$ & $0.05 \pm 0.08$ & $-0.17 \pm 0.05$ \\
\hline
\end{tabular}

${ }^{\text {a }}$ After M. J. Person et al. (2007, in preparation).

b In Pluto's atmosphere (not the shadow).

c At the half-light radius.

${ }^{\mathrm{d}}$ For an $\mathrm{N}_{2}$ atmosphere.

e The weighted average of the temperatures for all 3 years is $102 \pm 4 \mathrm{~K}$, and the weighted average of the temperature gradients is $-0.11 \pm 0.04 \mathrm{~K} \mathrm{~km}^{-1}$. 


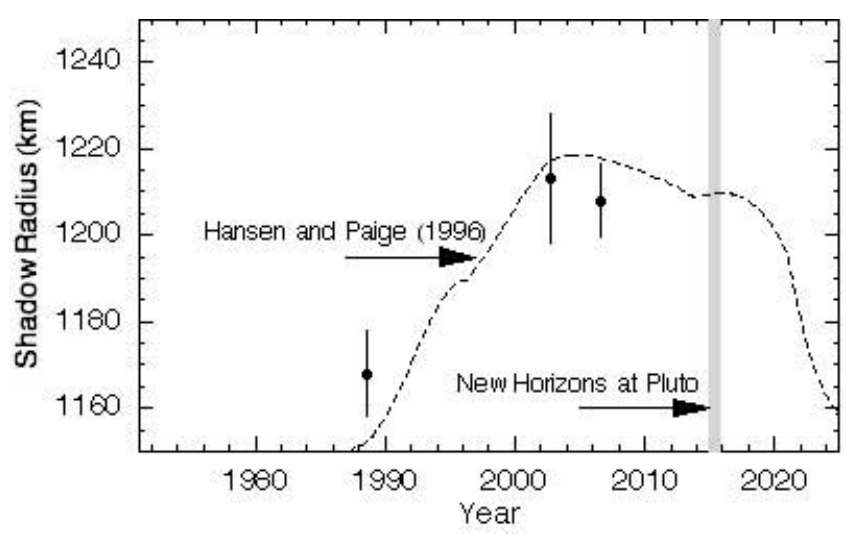

FIG. 7.- Half-light shadow radius of Pluto's atmosphere vs. year of observation. The points represent the half-light radius of Pluto's atmosphere (in the shadow plane) measured during each of the three observed stellar occultations by Pluto. The 1988 June shadow radius is a preliminary result, based on a reanalysis of the 1988 data with the position of the KAO as a free parameter (M. J. Person et al. 2007, in preparation). The 2002 August point is from Elliot et al. (2003b; see the caption to their Fig. 1). The present result for the 2006 data is plotted with an error bar of $\pm 10 \mathrm{~km}$ to allow for systematic error; in Table 5 we see that the halflight shadow radius derived from the fit 4 results is $10 \mathrm{~km}$ greater than the halflight shadow radius derived from the adopted fit 2 results, indicating a possible systematic error of this magnitude. The dashed line shows the half-light shadow radius derived from a frost-migration model selected from those published by Hansen \& Paige (1996; shown by "Run 31" in their Fig. 7). Since Hansen \& Paige (1996) calculated surface pressure, we derived the shadow radius from their model by assuming a mean scale height of $50 \mathrm{~km}$ between the surface and the shadow radius. The thermal inertia of the surface in this model is $1 \times 10^{3} \mathrm{cal} \mathrm{K}^{-1}$ $\mathrm{cm}^{-2} \mathrm{~s}^{-1 / 2}$, a value at the low end of the range considered by Hansen \& Paige (1996). The surface radius was set at $1173 \mathrm{~km}$ so that the frost-migration model would approximately match the three data points; even better agreement could likely be achieved by adjustment of the other parameters of the model.

"Run 31 " shown in their Fig. 7) that matches the data reasonably well. Since Hansen \& Paige (1996) give surface pressure versus time, we have to invoke an atmospheric model between the halflight radius and the surface in order to compare the occultation results with their models. However, the atmospheric structure between the half-light radius and the surface is highly uncertain. Since we are interested mainly in changes to the surface pressure, we have little choice but to make the assumption that the atmospheric structure remained the same between the half-light radius and the surface over the time period of interest (19882006). This assumption, of course, may be wrong, since we know that the shape of the occultation light curve changed (Fig. 6). Proceeding with this assumption as the approach we must take for now, an improper choice of model means only that we will infer the wrong surface radius, but the changes will be valid. Therefore, we assume a mean scale height of $50 \mathrm{~km}$, and we extrapolate this scale height downward until we reach the surface pressure of the Hansen $\&$ Paige (1996) model. We use this surface radius $(1173 \mathrm{~km})$ for the other years and use the atmospheric structure originally assumed to calculate the shadow radius at half-light. This is what is plotted in Figure 7 as the dashed line representing the Hansen \& Paige (1996) model. This approach assumes that we have a deep troposphere, and any changes in surface pressure cause the atmosphere to expand and contract the troposphere. On the other hand, if the atmosphere were to expand and contract where the atmospheric scale height is larger, then we would infer a different surface radius. When we better understand the atmospheric structure between the half-light radius and the surface, we can improve the inference of surface pressure from knowledge of the shadow halflight radius.

The shadow radius of Pluto's atmosphere at the half-light level for the 1988 data of $1154 \pm 20 \mathrm{~km}$, given in the caption of Figure 1 of Elliot et al. (2003b), was based on the astrometric solution of Elliot et al. (1989) rather than the more extensive astrometric solution of Millis et al. (1993), which yields a shadow radius of $1175 \pm 20 \mathrm{~km}$. The main source of the large error is the uncertain position of the KAO. In order to obtain a more certain result, we have applied our new analysis method to the 1988 data (M. J. Person et al. 2007, in preparation), allowing the position of the KAO in $(f, g)$ space to be fit. We find a preliminary result for the half-light radius of Pluto's shadow of $1168 \pm 10 \mathrm{~km}$, which we have plotted in Figure 7. This result is similar to that of Millis et al. (1993), but the uncertainty in the KAO position is no longer an issue.

Figure 7 shows that the shadow radius increased between 1998 and 2002, but it exhibited no change between 2002 and 2006 within the uncertainties of the data. This result agrees with the frost-migration models having lower thermal inertias. These models predict that substantial atmosphere will be available for study by the New Horizons spacecraft when it flies by Pluto in 2015. According to the frost-migration model displayed, a decrease in the shadow radius of Pluto's atmosphere should be noticeable from occultation observations (of similar quality to the ones described here) made after $\sim 2020$.

\section{CONCLUSIONS AND FUTURE WORK}

Over the 18 year span between 1988 and 2006, during which we have probed Pluto's atmosphere three times with multistation occultation observations, the structure of the atmosphere above a radius of $\sim 1230 \mathrm{~km}$ has not changed, with a temperature of $\sim 100 \mathrm{~K}$ and a small decrease of temperature with increasing altitude. This region can be described by current physical models. In contrast with this, Pluto's atmosphere below $\sim 1230 \mathrm{~km}$ cannot be described by current physical models and showed remarkable structural changes during the 14 years between 1988 and 2002. During the following 4 years, between 2002 and 2006, this portion of the atmosphere revealed only subtle changes in structure.

The evolution of the shape of the occultation light curve between 1988 and 2006 has led us to hypothesize ( $(6.3 .1)$ that a process causing substantial extinction in Pluto's lower atmosphere occurred before 1988 June, and that the atmosphere has since been clarifying. If this hypothesis is true, there also might be a signature of the changing extinction in the photometric record of reflected light from Pluto-Charon over the years, but it would be entwined with the ever-changing aspect at which we view the system.

Inversion, under the assumption of a clear atmosphere, combined with constraints imposed by atmospheric structure, sets the allowed range for the surface radius to be between 1120 and $1184 \mathrm{~km}$. The corresponding bulk densities of Pluto would be 2.22 and $1.88 \mathrm{~g} \mathrm{~cm}^{-3}$, which can be compared with Triton's density of $2.06 \pm 0.01 \mathrm{~g} \mathrm{~cm}^{-3}$ (McKinnon et al. 1995). If the two bodies have equal densities, then Pluto's radius would be $1148 \mathrm{~km}$.

We have demonstrated that our inversion temperature profile closely matches one of the model runs published by Strobel et al. (1996). This suggests that, contrary to the case in 1988, the current shape of Pluto's occultation light curve is not so extreme that it cannot be reproduced by a physical model containing only heat conduction and radiative transfer involving trace amounts of $\mathrm{CH}_{4}$ and $\mathrm{CO}$. Establishing the precise ranges of $\mathrm{CH}_{4}$ and $\mathrm{CO}$ abundances for which the Strobel et al. (1996) model matches the data should prove to be a fruitful exercise. The atmospheric abundances can then be used to constrain models of the surfaceice distribution that have been developed by detailed modeling of spectra (Olkin et al. 2007).

Although we have shown a range of temperature profiles for the deep-troposphere scenario, we have not studied which of 
these would be consistent with our current light curves in terms of any subtle starlight - or not so subtle starlight, such as lightcurve caustics - from the far limb (Stansberry et al. 1994). This work would establish firmer limits on the existence of a troposphere and the allowed range for the surface radius. Definitive results, however, must await higher $\mathrm{S} / \mathrm{N}$ light curves that probe closer to the center of the occultation shadow, which may reveal the signature of an occultation by the surface. NASA's Stratospheric Observatory for Infrared Astronomy (SOFIA; Becklin 1997) will make this goal easier to achieve when it becomes operational in 2010.

Although Pluto's atmosphere should ultimately begin collapsing as it recedes from the Sun, the frost-migration model (Hansen \& Paige 1996) that matches our data predicts that there will be a substantial atmosphere when the New Horizons mission reaches Pluto and its satellites in 2015 (Stern \& Spencer 2003).

Spectra of Eris and $2005 \mathrm{FY}_{9}$-Kuiper Belt objects about the size of Pluto and Triton-reveal $\mathrm{CH}_{4}$ ice on their surfaces (Brown et al. 2005; Licandro et al. 2006). Hence, these bodies may have an inventory of ices similar to Pluto and Triton and have atmospheres as well. When these bodies are sufficiently close to the Sun, such atmospheres could be dense enough to be detectable with the stellar-occultation technique (Elliot \& Kern 2003).

We thank Ian Bedford, Lynton Hemer, and Fraser Farrell at the Star Castle Observatory (Black Springs, South Australia), and Ian Ritchie of Electro Optic Systems (Mount Stromlo, Canberra, ACT, Australia) for telescope and observing support. We also thank Ted Dunham, Brian Taylor, Ralph Nye, and Rich Oliver for setting up and maintaining the Astrograph and CCD system at Lowell Observatory during prediction observations. The referee, John Stansberry, provided many helpful comments that improved the paper, and we are very grateful for his efforts. Financial support for Mount Canopus Observatory was provided by Williams College and David Warren. This work was supported in part by NASA Planetary Astronomy, through grants NNG 04-GE48G, NNG 04-GF25G, and NNH 04-ZSS001N to MIT and Williams College.
Becklin, E. E. 1997, in The Far Infrared and Submillimetre Universe, ed. A. Wilson (Noordwijk: ESA), 201

Binzel, R. P., \& Hubbard, W. B. 1997, in Pluto and Charon, ed. S. A. Stern \& D. J. Tholen (Tucson: Univ. Arizona Press), 85

Brown, G. N., Jr., \& Ziegler, W. T. 1980, Adv. Cryog. Eng., 25, 662

Brown, M. E., Trujillo, C. A., \& Rabinowitz, D. L. 2005, ApJ, 635, L97

Elliot, J. L., Dunham, E. W., Bosh, A. S., Slivan, S. M., Young, L. A., Wasserman, L. H., \& Millis, R. L. 1989, Icarus, 77, 148

Elliot, J. L., \& Kern, S. D. 2003, Earth Moon Planets, 92, 375

Elliot, J. L., \& Olkin, C. B. 1996, in Annual Review of Earth and Planetary Sciences, ed. G. W. Wetherill, A. L. Albee, \& K. C. Burke (Palo Alto: Annual Reviews), 89

Elliot, J. L., Person, M. J., \& Qu, S. 2003a, AJ, 126, 1041

Elliot, J. L., Strobel, D. F., Zhu, X., Stansberry, J. A., Wasserman, L. H., \& Franz, O. G. 2000, Icarus, 143, 425

Elliot, J. L., \& Young, L. A. 1992, AJ, 103, 991

Elliot, J. L., et al. 1993, AJ, 106, 2544

2003b, Nature, 424, 165

Eshleman, V. R. 1989, Icarus, 80, 439

Gulbis, A. A. S., et al. 2006, Nature, 439, 48

Gurwell, M. A., \& Butler, B. J. 2005, BAAS, 37, 743

Hansen, C. J., \& Paige, D. A. 1996, Icarus, 120, 247

Hubbard, W. B., Hunten, D. M., Dieters, S. W., Hill, K. M., \& Watson, R. D. 1988, Nature, 336, 452

Hubbard, W. B., Yelle, R. V., \& Lunine, J. I. 1990, Icarus, 84, 1

Krasnopolsky, V. A., \& Cruikshank, D. P. 1999, J. Geophys. Res., 104, 21979

Lellouch, E. 1994, Icarus, 108, 255

Licandro, J., Pinilla-Alonso, N., Pedani, M., Oliva, E., Tozzi, G. P., \& Grundy, W. 2006, A\&A, 445, L35

McDonald, S. W., \& Elliot, J. L. 2000, AJ, 119, 1999 (erratum 120, 1599)

McKinnon, W. B., Lunine, J. I., \& Banfield, D. 1995, in Neptune and Triton, ed. D. P. Cruikshank (Tucson: Univ. Arizona Press), 807

Millis, R. L., et al. 1993, Icarus, 105, 282
Nicholson, P. D., McGhee, C., \& French, R. G. 1995, Icarus, 113, 57

Olkin, C. B., et al. 2007, AJ, 133, 420

Owen, T. C., et al. 1993, Science, 261, 745

Pasachoff, J. M., et al. 2005, AJ, 129, 1718 2006, AAS DPS Meeting, 38, 25.02

Person, M. J., Elliot, J. L., Gulbis, A. A. S., Pasachoff, J. M., Babcock, B. A., Souza, S. P., \& Gangestad, J. W. 2006, AJ, 132, 1575

Reinsch, K., Burwitz, V., \& Festou, M. C. 1994, Icarus, 108, 209

Sicardy, B., et al. 2003, Nature, 424, 168

Soderblom, L. A., et al. 1990, Science, 250, 410

Souza, S. P., Babcock, B. A., Pasachoff, J. M., Gulbis, A. A. S., Elliot, J. L., Person, M. J., \& Gangestad, J. W. 2006, PASP, 118, 1550

Stansberry, J. A., Lunine, J. I., Hubbard, W. B., Yelle, R. V., \& Hunten, D. M. 1994, Icarus, 111, 503

Stansberry, J. A., Lunine, J. I., \& Tomasko, M. G. 1989, Geophys. Res. Lett., 16,1221

Stern, S. A., \& Spencer, J. 2003, Earth Moon Planets, 92, 477

Strobel, D. F., Zhu, X., Summers, M. E., \& Stevens, M. H. 1996, Icarus, 120, 266

Tholen, D. J., \& Buie, M. W. 1990, BAAS, 22, 1129

1997, in Pluto and Charon, ed. S. A. Stern \& D. J. Tholen (Tucson: Univ. Arizona Press), 193

Tryka, K. M., Brown, R. H., Cruikshank, D. P., Owen, T. C., Geballe, T. R., \& DeBergh, C. 1994, Icarus, 112, 513

Yelle, R. V., \& Elliot, J. L. 1997, in Pluto and Charon, ed. S. A. Stern \& D. J. Tholen (Tucson: Univ. Arizona Press), 347

Yelle, R. V., \& Lunine, J. I. 1989, Nature, 339, 288

Young, E. F., \& Binzel, R. P. 1994, Icarus, 108, 219

Young, L. A., Elliot, J. L., Tokunaga, A., de Bergh, C., \& Owen, T. 1997, Icarus, 127, 258

Zacharias, N., Urban, S. E., Zacharias, M. I., Wycoff, G. L., Hall, D. M., Monet, D. G., \& Rafferty, T. J. 2004, AJ, 127, 3043 